\title{
Disseminated cryptococcosis in a deceased with HIV-1 diagnosed by Minimally Invasive Tissue Sampling Technique
}

\author{
Nuwadatta Subedi ${ }^{1}$, Suraj Bhattarai ${ }^{1}$, Sunita Ranabhat ${ }^{1}$, Binita Sharma ${ }^{1}$, Madan Baral ${ }^{2}$, \\ and Tirtha Upadhyaya ${ }^{1}$ \\ ${ }^{1}$ Gandaki Medical College Teaching Hospital and Research Center Pvt Ltd \\ ${ }^{2}$ Pokhara Academy of Health Sciences
}

December 30, 2020

\begin{abstract}
Cryptococcosis is one of the common mycological conditions leading to morbidity and mortality in patients with AIDS. We are presenting a case of disseminated cryptococcosis in a patient infected with human immunodeficiency virus (HIV-1), which were both diagnosed postmortem using Minimally Invasive Tissue Sampling Method.
\end{abstract}

\section{Introduction}

Cryptococcosis is one of the common mycological condition leading to morbidity and mortality in patients with AIDS. ${ }^{1}$ Cryptococcus can cause life threatening infections like meningoencephalitis and disseminated cryptococcosis in immunocompromised patients. ${ }^{2}$ According to the studies, 10 to $25 \%$ of AIDS patients with cryptococcosis die even if they have received antifungal therapy and 30 to $60 \%$ die within the first year of onset of the infection. ${ }^{1}$

We present a case of disseminated cryptococcosis in a patient with HIV, which were both diagnosed by clinical autopsy using Minimally Invasive Tissue Sampling (MITS) technique. ${ }^{3}$ In Nepal, clinical autopsies are not usually conducted and forensic autopsies are not routinely backed up with histopathological, microbiological and other ancillary tests. In this case, we performed MITS procedure along with histopathological, serological and microbiological tests as a part of a research project - Determining Efficiently the Cause of Death among Adults and Generating Mortality Evidence at MITS Alliance Unit Nepal (DECODE MAUN Nepal).

\section{Case details}

A 35 years old female was allegedly having myalgia since five days and was taking analgesics as selfmedication. She was found dead at her own home after few days of the onset of her symptoms. As the case was a sudden death, it was reported to the law enforcement agencies and after necessary inquest, the deceased was brought to our study site for medico legal autopsy. According to her relatives, the deceased was married, housewife, living with her two children and was not reported to be having any underlying systemic disease or condition during her lifetime. There was denial of any significant trauma during her life. She was non-smoker, non-user of smokeless tobacco and non-alcoholic.

On external examination, there were ill-defined contusions on the subject's periorbital region, $5 \mathrm{X} 3 \mathrm{~cm}$ contusions on both the knees, and diffuse $6 \mathrm{X} 7 \mathrm{~cm}$ contusion in the right thigh. These contusions could be attributed to her staggering efforts to move around in the last few days of her life. There was clotted blood in the perineal and vaginal region, but no injuries were evident; it was consistent with menstrual bleed confirmed by the statements from her family members. On complete autopsy examination, no obvious internal injuries were detected. Both the lungs were mildly edematous with patches of consolidation. The spleen was 
grossly enlarged to approximately one kilogram in weight and $30 \mathrm{X} 25 \mathrm{~cm}$ in size. The parenchyma of the spleen was heavily congested.

We collected $20 \mathrm{ml}$ of blood from the subject's subclavian vein and $10 \mathrm{ml}$ of cerebrospinal fluid (CSF) from the occipital puncture. We collected tissue samples using MITS technique from the subject's key organs: brain tissue sample through occipital and trans nasal approach, right and left lung tissue, and liver tissue samples. Additionally, we collected spleen tissue as it was grossly enlarged, and rectal swab. The first samples from both the right and left lungs were collected using a new sterile needle and placed into Brain Heart Infusion (BHI) broth for culture, then incubated for 72 hours. The subsequent lung and other tissue samples were placed into labeled tissue cassettes, which were subsequently placed into a tissue jar containing $10 \%$ neutral buffered formalin solution and then sent for histopathological examination. Initially the tissue sections were reviewed by Hematoxylin and Eosin $(\mathrm{H} \& \mathrm{E})$ stains and later analyzed by Periodic Acid-Schiff (PAS).

About $3 \mathrm{ml}$ sample of blood was kept into BHI broth and incubated for seven days. Microscopic examination of CSF sample by Gram's stain and India Ink preparations was performed. CSF samples were cultured in Blood agar (BA) and Chocolate agar (CA) plates and incubated at $35^{\circ} \mathrm{C}$ for seven days. CSF samples were incubated in two tubes of Sabouraud dextrose agar (SDA); one tube was incubated at $35^{\circ} \mathrm{C}$ and another tube at room temperature. Urea hydrolysis test was performed from the colonies obtained from BA, CA and SDA.

\section{Results}

Histopathological findings (Figure 1): Alveolar spaces of both the right and left lungs were filled with round refractile structures with thick capsule suggestive of cryptococci. There were also few alveolar macrophages (Figure 1A and 1B). There were similar structures suggestive of cryptococci in brain tissues too (Figure 1C). Liver was unremarkable, whereas spleen had areas of extensive congestion along with dense infiltration by cryptococci (Figure 1D).

Peripheral blood smear was negative for malarial parasites. Serology was positive for HIV-1 antibodies by HIV-Tri Dot assay but the serological tests for dengue, malaria, HBsAg, HCV, Brucella and Leptospira were all negative. Microbiological findings (Figure 2): Gram stain of CSF sample revealed Gram positive round yeast cells suggestive of Cryptococcus spp (Figure 2A). Typical round encapsulated yeast forms suggestive of Cryptococcus spp were demonstrated by India ink preparation of CSF (Figure 2B). Colonies in Sabouraud dextrose agar were creamy, white and mucoid. Similarly, colonies on blood agar, chocolate agar and Sabouraud dextrose agar showed gram positive round budding yeast cells suggestive of Cryptococcus spp . Urease test was also positive after 48 hours of incubation. The most remarkable finding was Cryptococcus neoformans complex organism detected by CSF culture. Additionally, the lung tissue culture showed the growth of

\section{Proteus miribilis.}

The cause of death was determined according to the World Health Organization International Classification of Diseases (ICD 10) protocol and based on the consensus of 'Cause of Death' panel within our research project. The immediate cause of death in this case (Part IA) was disseminate cryptococcosis (ICD code B45.7) with underlying HIV/AIDS - ICD B20.5 (Part IB). Pneumonia due to Proteus mirabilis (J15.6) was the secondary condition (Part II) which was not directly related to the death.

\section{Discussion}

We have presented a case of disseminated cryptococcosis in a HIV infected female of 35 years age. Neither HIV nor cryptococcosis had been diagnosed during her lifetime. Therefore, our finding highlights the importance of performing proper laboratory and pathology investigations at the time of autopsy to determine the cause of death. In Nepal and in many low-and-middle income countries, clinical autopsies are usually not conducted, so the cause of death remains undiagnosed in many cases. Forensic autopsies are conducted only when there is an official request from the Law enforcement agencies, mostly in unnatural and sudden or suspicious 
deaths. Even forensic autopsies are not routinely backed up with ancillary investigations which leads to undetermined cause of deaths. In such cases, histopathological, microbiological and other relevant tests in human specimens could have contributed to establish the cause of death. From microbiological perspective, this gap in evidence indicates the need for taking proper precautions to potentially contagious infections like HIV during an autopsy and regard every case as potentially infectious unless proven otherwise. The diagnosis of HIV in the married female with two children can have significant implications to the family members, as the husband could have also contracted the disease and there could also have been vertical transmission to her children too. In this context, our team has counselled the family members to perform HIV test and seek treatment if needed according to the protocol. The condition of disseminated cryptococcosis implies that the deceased could be in immunocompromised state for a long time.

The periorbital contusions and the contusions on the thighs in the absence of any internal injuries rules out significant trauma. Gross examination during the autopsy failed to attribute the cause of death, but the ancillary investigations were useful to establish disseminated cryptococcosis as the final or direct cause of death with HIV/AIDS as underlying cause. Cryptococcus causes meningoencephalitis and disseminated cryptococcosis usually in immunocompromised hosts, ${ }^{4,5}$ though cases of cryptococcal meningitis have also been reported in immunocompetent patients. ${ }^{6-8}$ The research on the prevalence of cryptococcosis is very sparse in literature from Nepal. In a record review of one fiscal year in the major tertiary center of Nepal, among 15 patients with Cryptococcal meningoencephalitis, the majority $(9,60 \%)$ had HIV infection. ${ }^{5}$

The involvement of cryptococcus in causing death among HIV infected patients was demonstrated in approximately $10 \%$ cases by an autopsy study in low-income settings in Mozambique. ${ }^{9}$ The pathology of cryptococcal meningoencephalitis with minimal inflammatory infiltrates and gelatinous pseudocysts produced by abundant C. neoformans was shown in most of the cases in an autopsy study in HIV-infected patients. ${ }^{1}$

There are several ways of diagnosing cryptococcosis. CSF culture is regarded as gold standard method and microscopy can be added to aid in the diagnosis. In the recent years, there has been evolution of cryptococcal antigen $(\mathrm{CrAg})$ testing which has proven to be relatively inexpensive and more sensitive method. In order to facilitate easy visualization of the fungus in the specimens, stains like India ink are also used. ${ }^{11}$ The India ink staining for fungus detected cryptococcus in our case as well. The sensitivity of India ink staining to detect cryptococcus is up $80 \%$ in HIV-positive patients. ${ }^{12}$

Postmortem examination should be done meticulously, and the use of ancillary tests should be made available to attribute mortality to specific pathogens. The accurate diagnosis of cause of death is important as it informs the burden of disease in the region and supports the surveillance system of the country. Moreover, it can make a great impact to public health policies and helps in the prophylaxis and treatment modalities, particularly in infectious diseases.

\section{Conclusion}

Disseminated cryptococcosis was attributed to the final cause of death in the deceased with underlying HIV disease, who was not diagnosed with either of these conditions during her lifetime. Clinical autopsy should be conducted meticulously along with adequate ancillary tests to correctly identify the cause of death as it can provide valuable information about disease burden in the region. Minimal invasive tissue sampling technique can be a useful tool in determining cause of death in low resource settings. In mortality cases with likely infectious conditions and other natural diseases, clinical autopsies have an important clinical and public health implications.

\section{Acknowledgments}

We would like to thank the MITS Alliance team for their continuous guidance and support in our surveillance study. We would also like to acknowledge our study sites (Gandaki Medical College, Pokhara Academy of Health Sciences, Damauli Hospital) for their cooperation in research activities. Finally, we thank relatives of the case for providing case-related information even at the time of grief and sorrow.

Conflict of interest : This is one of the cases as a part of a research project - Determining Efficiently the 
Cause of Death among Adults and Generating Mortality Evidence at MITS Alliance Unit Nepal (DECODE MAUN Nepal) supported by MITS Surveillance Alliance.

\section{Author Contributions:}

NS performed MITS, participated in cause of death determination and prepared the first draft of the manuscript. SB reviewed clinical information of the case, participated in cause of death determination and contributed to manuscript writing. SR reported Pathological tests and participated in cause of death determination and reviewed the draft of the manuscript. BK reported microbiological tests and participated in cause of death determination and reviewed the draft of the manuscript. MPB performed MITS, participated in cause of death determination and reviewed the draft of the manuscript. TLU reviewed clinical information of the case, participated in case discussion for cause of death determination and reviewed the draft of the manuscript.

Data availability statement: Data sharing is not applicable to this article as no datasets were generated or analyzed from this single case.

Informed consent: Written informed consent was taken from the nearest family member of the deceased for publishing the case details and associated laboratory images.

\section{Funding:}

The work is supported by the MITS Surveillance Alliance.

The MITS Surveillance Alliance is funded by the Bill and Melinda Gates Foundation- Opportunity/Contract ID: OPP1180554

\section{References:}

1. Jarvis JN, Harrison TS. HIV-associated cryptococcal meningitis.AIDS . 2007;21(16):2119-2129. doi:10.1097/QAD.0b013e3282a4a64d

2. Maziarz EK, Perfect JR. Cryptococcosis. Infect Dis Clin North Am . 2016;30(1):179-206. doi:10.1016/j.idc.2015.10.006

3. What Is MITS - MITSPortal. https://mitsalliance.org/WhatIsMITS. Accessed November 8, 2020.

4. Aldhaleei WA, Bhagavathula AS, Alhajeri A. Vision loss in an AIDS patient with cryptococcal meningitis. Med Mycol Case Rep . 2019;24(April):51-53. doi:10.1016/j.mmcr.2019.04.001

5. Kharel G, Ojha R. Spectrum of Cryptococcal Meningoencephalitis in Tertiary Hospital in Nepal Spectrum of Cryptococcal Meningoencephalitis in Tertiary Hospital in Nepal. Journal of Institute of Medicine.2018;40(2):27-32.

6. Jha A, Adhikari S, Sigdel KR, et al. Case report: Cryptococcal meningitis in an apparently immunocompetent patient in nepal-challenges in diagnosis and treatment. Wellcome Open Res . 2019;4:1-16. doi:10.12688/wellcomeopenres.15187.2

7. Khanal B, Sharma SK DM. Cryptococcal Meningitis in a non-AIDS patient. J Nepal Med Assoc . 2003;41(142):323-325. doi:https://doi.org/10.31729/jnma.759

8. Radheshyam KC, Karkey A, Prajapati KG, Baker S, Basnyat B. Fatal cryptococcal meningitis in a HIVseronegative patient with liver cirrhosis. JMM Case Reports . 2014;1(3):10-12. doi:10.1099/jmmcr.0.001982

9. Hurtado JC, Castillo P, Fernandes F, et al. Mortality due to Cryptococcus neoformans and Cryptococcus gattii in low-income settings: an autopsy study. Sci Rep . 2019;9(1):1-10. doi:10.1038/s41598-019-43941-w

10. Klock C, Cerski M, Goldani LZ. Histopathological aspects of neurocryptococcosis in HIV-infected patients: autopsy report of 45 patients. Int J Surg Pathol . 2009;17(6):444-448. doi:10.1177/1066896908320550 
11. Nalintya E, Kiggundu R, Meya D, Road MH. Evolution of Cryptococcal Antigen Testing : What is new ? 2017;10(2):62-67. doi:10.1007/s12281-016-0256-3.Evolution

12. O'Halloran JA, Powderly WG, Spec A. Cryptococcosis Today: It Is Not All About HIV Infection. Curr Clin Microbiol Reports . 2017;4(2):88-95. doi:10.1007/s40588-017-0064-8

\section{Figure captions:}

Figure 1. Histopathological findings of tissue specimens collected by MITS technique. A) Right Lung with cryptococci in alveolar spaces (H\&E 40X); B) Left Lung with cryptococci in alveolar spaces (H\&E 40X); C) Brain tissue with cryptococci (PAS Stain 40X); D) Spleen with dense infiltration with cryptococci (PAS stain 10X)

Figure 2. Microbiological findings of CSF specimen. A) Gram's stain showing Gram-positive budding round yeast cells suggestive of Cryptococcus spp ; B) Indian ink staining showing capsule of

Cryptococcus spp and the zone of clearance or 'halo' surrounding the yeast cells
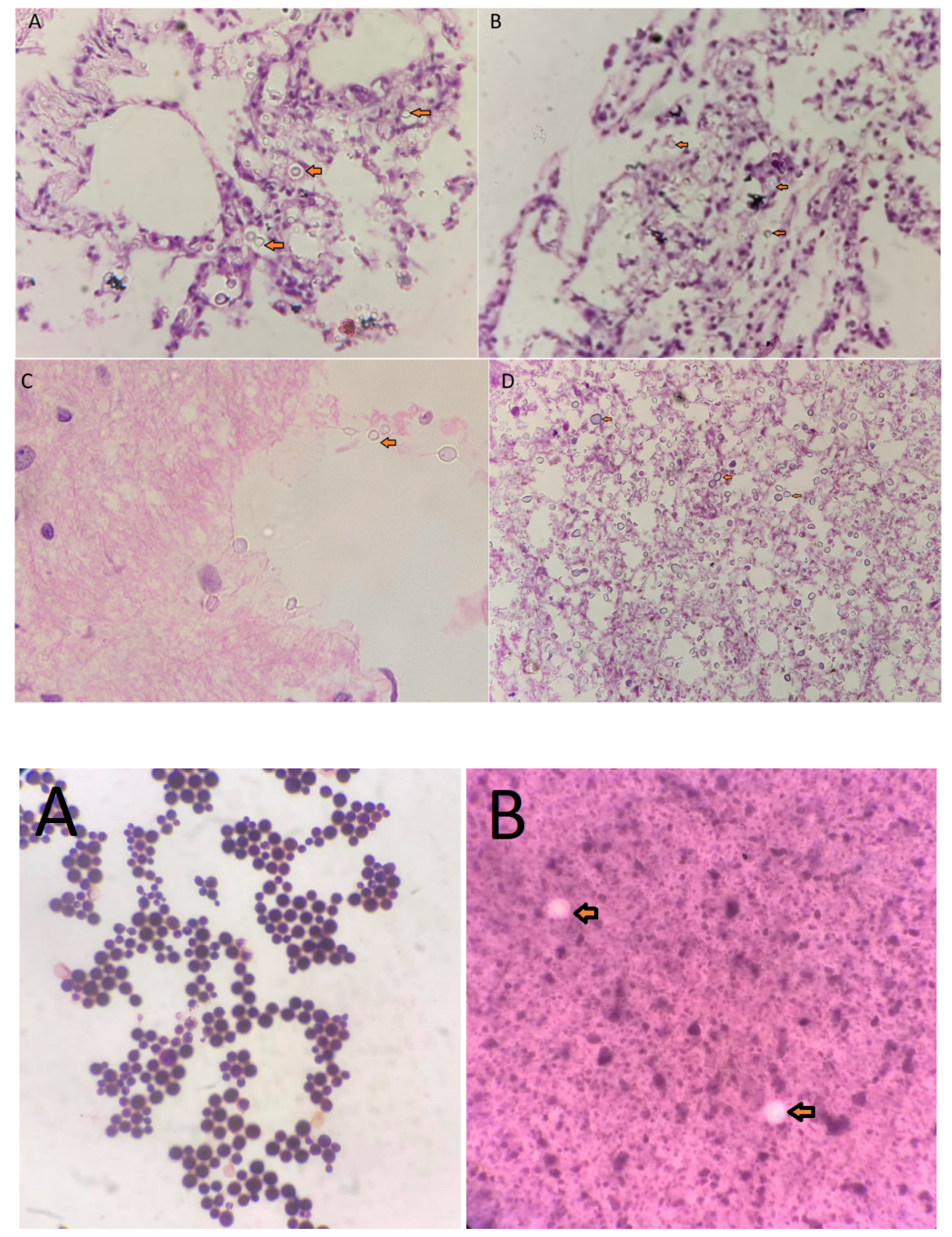